\title{
THE EFFECTS OF INFUSION WATER TYPE AND FERMENTATION TIME ON MOSQUITO AND NON-TARGET ORGANISM COLLECTED IN THE CDC'S AUTOCIDAL GRAVID OVITRAP
}

\author{
LAGAN J. MULLIN, DANIEL DIXON'AND RUI-DE XUE \\ Anastasia Mosquito Control District, 120 EOC Drive, St. Augustine, FL 32092 \\ Current Address: USDA/CMAVE, 1600-1700 SW 23 $3^{\text {rd }}$ Dr. Gainesville, FL 32608 \\ Guest Editor: Caroline Efstathion
}

\begin{abstract}
The Autocidal Gravid Ovitrap (AGO) is being marketed as an alternative mosquito control tactic to potentially harmful spraying. One problem is the high number of non-target organisms captured with AGOs that is not present in other gravid traps utilized by mosquito control districts. We tested three different infusion water types with a tap water control in AGOs to determine if they would reduce non-target capture rates. However, all three infusion water types captured more non-target organisms than the tap water control, and the infusion water did not have a significant effect on the number of Aedes aegypti or Ae. albopictus mosquitoes collected. Fermentation time (the date) had an effect on non-target organism capture rates in the AGO traps, but weather conditions may have confounded the fermentation effect. Despite capturing a low number of mosquitoes, this trap was attractive to pest species such as Lucilia sericata. Overall, this trap was ineffective at capturing high rates of Aedes mosquitoes, but it may function as a passive pest control trap with future design modifications.
\end{abstract}

Key Words: Autocidal gravid ovitrap, Aedes aegypti, Aedes albopictus, infusion water, non-targets

\section{INTRODUCTION}

Aedes aegypti Linn. and Ae. albopictus Skuse are nuisance mosquitoes found in Florida and much of the United States that are known vectors of Dengue, Zika, Yellow Fever, Chikungunya and other arboviruses (WHO, 2012). These mosquitoes prefer to feed on people and rest near residential properties. In addition, Aedes aegypti and Ae. albopictus lay their eggs in bottles, plastic buckets, trash cans, and other artificial containers or ornamental plants associated with human housing (Barerra et al. 2006; Wilke et al. 2018; Hawley, 1988; Delatte et al. 2008; Wong et al. 2011).

One of the major obstacles to controlling Ae. aegypti is its propensity to oviposit in cryptic breeding sites (under housing structures with collected water, small bottle caps, hidden artificial containers, etc). Springstar developed the Biocare Autocidal Gravid Ovitrap (AGO), which captures and kills gravid Aedes females, as a way to control mosquitoes that are searching for these cryptic oviposition sites. The trap is based off the CDC Autocidal Gravid Ovitrap (Mackay et al. 2013), which consists of a five-gallon black bucket filled with infusion water that is modified to hold a capture chamber laced with a glue board. AGO traps were successful in Puerto Rico with a reported $60-80 \%$ reduction in female Ae. aegypti when used as part of an area-wide mosquito management strategy (Barrera et al. 2014). Ideally, autocidal gravid ovitraps will utilize the most effective infusion water to capture an abundance of a target mosquito species while minimizing bycatch. Previous studies determined that ovitrap capture rates for mosquitoes can be enhanced through the use of hay infusions rather than just water (Reiter et al. 1991; Trexler et al. 1998; Ponnusamy et al. 2010). For Ae. aegypti and Ae. albopictus, alfalfa (Montgomery et al. 2017), orchard grass (unpublished suggestion), and live oak (Ponnusamy et al. 2010) are the suggested infusion water substrates for ovitraps. However, the CDC Autocidal Gravid Ovitrap uses hay as its infusion water substrate (Bar- 
erra et al. 2014) which captures a high abundance of non-target organisms (Dixon et al. unpublished).

The purpose of this study was to compare the capture rates of mosquitoes and non-target organisms in AGO traps with alfalfa, orchard grass, hay and tap water infusions. The objective was to determine which infusion collected the lowest number of non-target organisms. This was a two-fold approach as both the type of infusion water substrate and its fermentation time were assessed. We hypothesize that both non-target and mosquito capture-rates will be highest after two weeks then begin to decline as the infusion water becomes too fermented to attract organisms. It is also expected that the most effective infusion-water substrate will be the hay utilized in the Springstar Biocare AGO trap.

\section{MATERIALS AND METHODS}

Autocidal Gravid Ovitraps were obtained from the company SpringStar (Woodinville, WA, U.S.A.) through an SBIR grant from the NIH (Grant \# 2R44AI115782-02) for testing the effectiveness of CDC AGO traps in the field. Infusion water was produced using 24 grams of the orchard grass, Springstar hay, or alfalfa and 8.5 liters of tap water. The control infusion was tap water only. These traps were deployed at the Evergreen Cemetery in St. Augustine Florida (29.894304, -81.335801) after each infusion set fermented in the buckets for one week.

Following a random block design with 4.6 meters between each trap and each block (no Latin shift rotations), nine traps filled with the different infusion water types and nine tap water controls were left in the field continuously for 6 weeks. The traps were arranged so one of each infusion water type and the tap water control were in different groups (9 groups of 4 traps). The tops of the AGO traps were replaced and brought back to the Anastasia Mosquito Control District (AMCD) Base station once a week for those six weeks to identify and record the number of mosquitoes and non-target organisms captured from each respective infusion water type.
Data was recorded in an excel spreadsheet to analyze mosquito and non-target collections in each AGO trap. A Kruskal-Wallis test was used to detect significant differences across the treatment conditions compared to the controls with a p-value $\leq 0.05$. This was followed up with a non-parametric comparison of all pairs using the Steel-Dwass Method. A generalized linear mixed model (GLMM) was used to determine if fermentation time influenced capture rate. Weather data was collected using Weather Underground (The Weather Company, San Francisco, CA) for the zip code of the study site. All statistical analyses were conducted through JMP software (Cary, NC, U.S.A.).

\section{RESULTS}

Overall, the infusions did not collect the number of mosquitoes that were expected. The number of Ae. aegypti and Ae. albopictus collected were very low and not statistically significant from the tap water control in all the infusions tested (Figure 1). However, the number of Culex captured with the Orchard grass and SpringStar Hay was greater than the control $\left(\chi_{(3)}^{2}=12.1, P=0.0071\right)$, but the orchard grass and springsSar hay collections of Culex were not significantly different from each other. In a similar fashion, the three infusions caught more non-targets than the control $\left(\chi_{(3)}^{2}=36.4, P<0.0001\right)$, but were not significantly different from each other.

The dataset for mosquitoes was Binomially distributed while the dataset for non-target organisms was Poisson distributed. According to the GLMM, the most important factor for the collection of mosquitoes (Figure $2)$ was the date $\left(P<0.0479, \chi_{(5)}^{2}=11.18\right.$, AICc $=217.3)$, specifically for weeks two through three of the study. For non-target organisms (Figure 3), the most important factors were the date $\left(P<0.0001, \chi_{(5)}^{2}=382.4\right)$ followed by the date and infusion $\left(P<0.0001, \chi_{(15)}^{2}\right.$ $=168.64)$, and finally the infusion $(P<0.0001$, $\left.\chi_{(3)}^{2}=168.3\right)$ all with an AICc $=3442.6$. The data collected from Weather Underground indicated that the rainfall during week three was $10.72 \mathrm{~cm}$, week one was $5.49 \mathrm{~cm}$, and the remaining weeks were all under $1.5 \mathrm{~cm}$. 


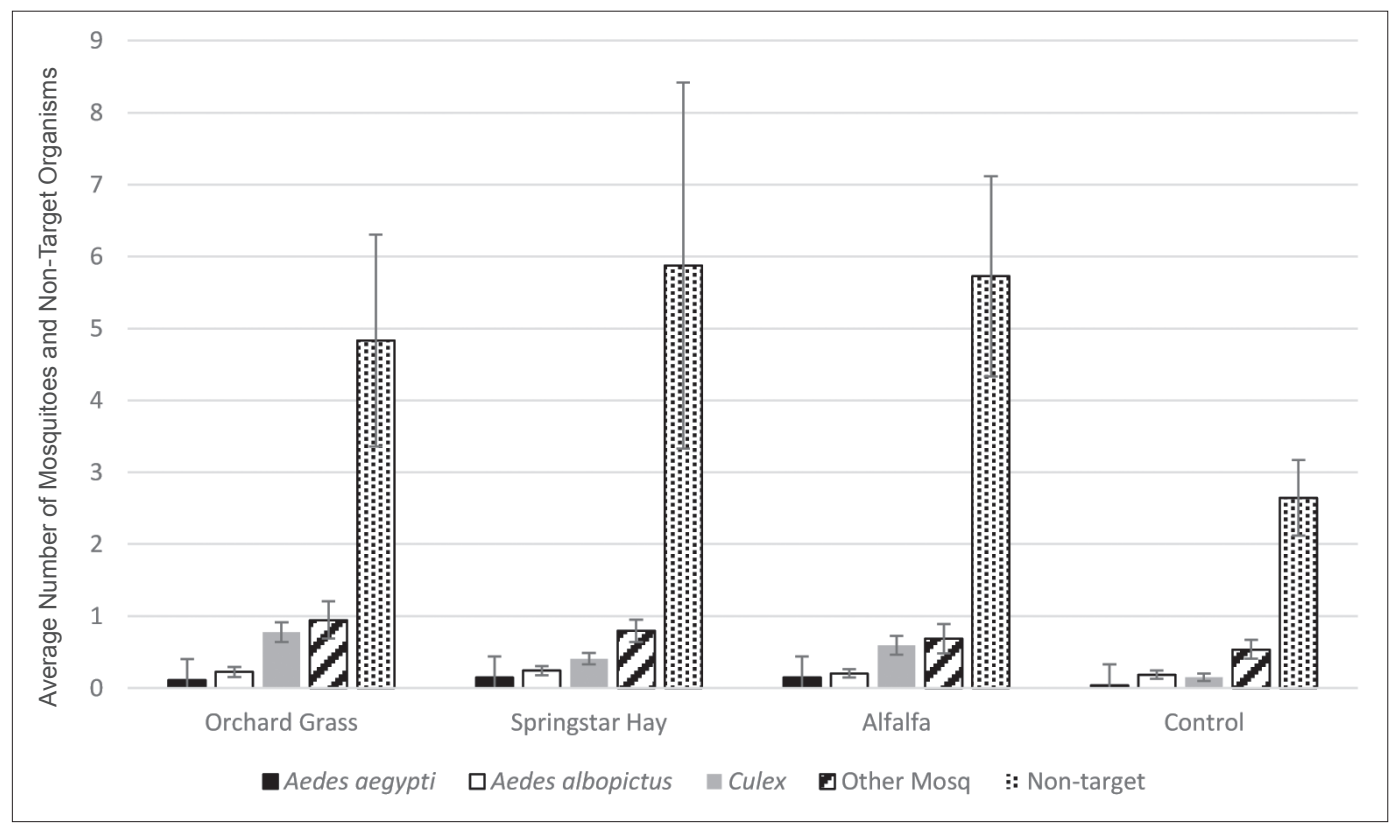

Figure 1. Effect of Infusion water type on mosquito and non-target organism capture rates. The X-axis shows the 3 different infusion water types (Orchard Grass, Springstar Hay, and Alfalfa) and the control (tap water). The Y-axis shows the average organism abundance. Error bars represent standard error (SE) of the mean.

\section{DISCUSSION}

Our study showed that all three infusion water types captured more non-target organ- isms than the tap water control, but the infusion water did not have a significant effect on the number of Ae. aegypti or Ae. albopictus mosquitoes collected. According to the

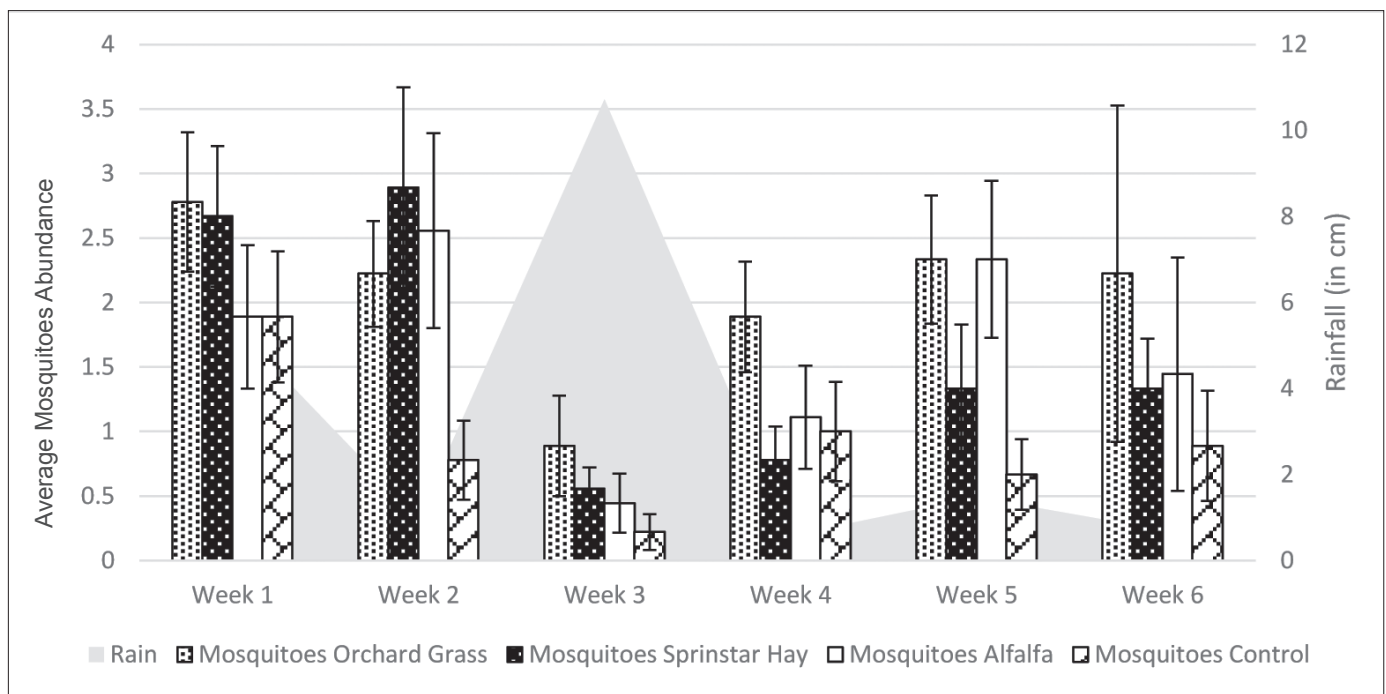

Figure 2. Effect of infusion water fermentation time on mosquito collections. The x-axis represents each week of the study. The primary y-axis (left side) represents mosquito abundance while the secondary y-axis (right side) represents rainfall in centimeters. The weekly rainfall is depicted as an area graph in the background in transparent grey. Each bar pattern represents a different infusion water type as shown in the legend above. Error bars represent standard error (SE). 


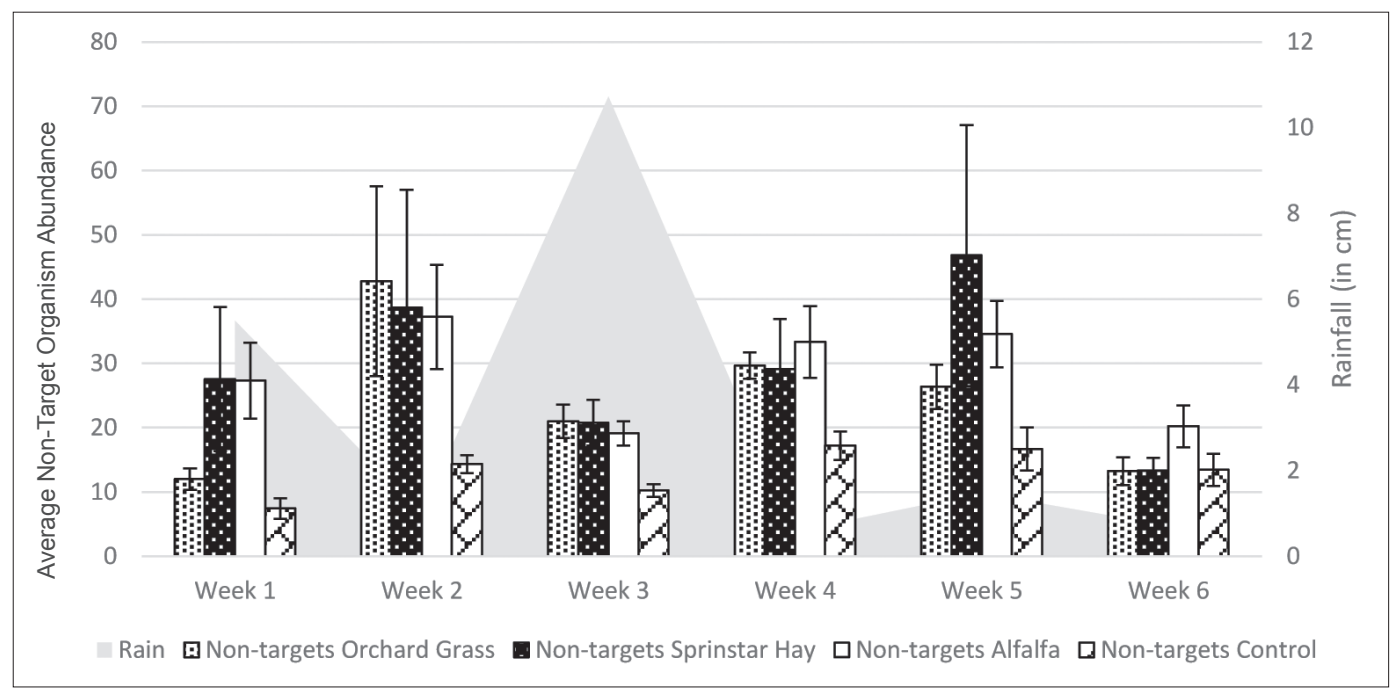

Figure 3. Effect of infusion water fermentation time on non-target collections. The x-axis represents each week of the study. The primary y-axis (left side) represents non-target organism abundance while the secondary y-axis (right side) represents rainfall in centimeters. The weekly rainfall is depicted as an area graph in the background in transparent grey. Each bar pattern represents a different infusion water type as shown in the legend above. Error bars represent standard error (SE).

GLMM, fermentation time (the date) had an effect on non-target organism capture rates in the AGOs, but weather conditions may have confounded the fermentation effect. Our results suggest that the infusion water types tested did not improve the efficacy of the CDC AGO.

As mentioned above, weather can be a major factor in field testing results. During week three of the study, just over $10.16 \mathrm{~cm}$ of rain was reported on Weather Underground which could explain the sudden decrease in populations of both mosquitoes and non-targets during that time. This decrease could have been attributed to an increase in alternative oviposition sites for mosquitoes and a possible dilution effect to the infusion water after the rain.

A large abundance of the mosquitoes captured were not Ae. aegypti and Ae. albopictus, which were the main target species of AGOs. The capture rates of Ae. aegypti and Ae. albopictus were quite low and not significantly different from each other. A majority of the mosquitoes captured were actually Culex (species not determined), Psorophora ferox, Ae. taeniorhynchus, and "other mosquitoes" some of which were unidentifiable. In addition to an abundance of non-target mosquitoes captured by the AGOs, on some trapping days an excess of over 200 Lucilia sericata were found in the AGOs. This was noticed in two different treatments, the alfalfa and orchard grass treatments. This is potentially due to the rotten fish smell exuded by the infusion water in the traps. $\mathrm{Lu}$ cilia sericata favor rotting flesh as a breeding site (Liu et al. 2016), therefore, the odorants coming from AGO infusions may function as attractants for this livestock pest species. Other non-target species captured by this trap ranged across multiple families and orders of organisms: Anolis carolinensis, Anolis sagrei, Lucilia sericata, Camponotus floridanus, Dyscinetus morator, Caenurgina erechtea, Campaea perlata, as well as various Chironomidae species.

The main goal of this project was to reduce the biological impact of AGOs on nontarget organisms. We can surmise from this study that infusion water substrate types were not one of the main attractants of Aedes mosquitoes to the AGOs in this study. However, the infusion water types were more attractive to non-target organisms. Although the Aedes mosquito capture rate was very low, AGOs have the potential to be modified as passive pest control traps. There are a vast array of 
agricultural and medical pest species that affect people. These species include Spodoptera frugiperda (moth), Anoplophora glabripennis (ant), Lucilia sericata (fly), and many others. Continued work with AGOs may facilitate a change in function of these traps to target other major medical and agricultural nuisance pests. Along those same lines, continued assessment of AGOs might divulge new practices to prevent any impact they might have on beneficial non-target organisms.

\section{REFERENCES CITED}

Barrea R, Amador M, Acevedo V, Hemme RR, Felix G. 2014. Sustained, area-wide control of Aedes aegypti using CDC autocidal gravid ovitraps. Am J Trop Med Hyg, 91: 1269-1276.

Barrera R, Amador M, Clark GG 2006. Ecological Factors Influencing Aedes aegypti (Diptera: Culicidae) Productivity in Artificial Containers in Salinas, Puerto Rico. Journal of Medical Entomology, 43: 484-492.

Delatte H, Dehecq JS, Thiria J, Domerg C, Paupy C, Fontenille D. 2008. Geographic Distribution and Developmental Sites of Aedes albopictus (Diptera: Culicidae) During a Chikungunya Epidemic Event. Vector-Borne and Zoonotic Diseases, 8: 25-34.

Hawley WA. 1988. The biology of Aedes albopictus. J Am Mosq Control Assoc Suppl, 1, 1-39.

World Health Organization. (2012). Global Strategy for Dengue Prevention and Control, 2012-2020 WHO, Geneva 2012.
Liu W, Longnecker M, Tarone A, Tomberlin J. 2016. Responses of Lucilia sericata (Diptera: Calliphoridae) to compounds from microbial decomposition of larval resources (Vol. 115).

Mackay AJ, Amador M, Barrera R. 2013. An improved autocidal gravid ovitrap for the control and surveillance of Aedes aegypti. Parasites Eं Vectors, 6: 225.

Montgomery BL, Shivas MA, Hall-Mendelin S, Edwards J, Hamilton NA, Jansen CC, et al. 2017. Rapid Surveillance for Vector Presence (RSVP): Development of a novel system for detecting Aedes aegypti and Aedes albopictus. PLOS Neglected Tropical Diseases, 11: e0005505.

Ponnusamy L, Xu N, Boroczky K, Wesson DM, Abu Ayyash L, Schal C, et al. 2010. Oviposition responses of the mosquitoes Aedes aegypti and Aedes albopictus to experimental plant infusions in laboratory bioassays. J Chem Ecol, 36: 709-719.

Reiter P, Amador MA, Colon N. 1991. Enhancement of the CDC ovitrap with hay infusions for daily monitoring of Aedes aegypti populations. J Am Mosq Control Assoc, 7: 52-55.

Trexler JD, Apperson CS, Schal C. 1998. Laboratory and field evaluations of oviposition responses of Aedes albopictus and Aedes triseriatus (Diptera: Culicidae) to oak leaf infusions. J Med Entomol, 35: 967-976.

Wilke ABB, Vasquez C, Mauriello PJ, Beier JC. 2018. Ornamental bromeliads of Miami-Dade County, Florida are important breeding sites for Aedes aegypti (Diptera: Culicidae). Parasit Vectors, 11: 283.

Wong J, Stoddard ST, Astete H, Morrison AC, Scott TW. 2011. Oviposition site selection by the dengue vector Aedes aegypti and its implications for dengue control. PLoS Negl Trop Dis, 5: e1015. 